\title{
Caracterização morfométrica de ovinos da raça Santa Inês criados nas microrregiões de Teresina e Campo Maior, Piauí
}

\section{Gregório da Silva Costa Júnior ${ }^{2}$, José Elivalto Guimarães Campelo ${ }^{3}$, Danielle Maria Machado Ribeiro Azevêdo ${ }^{4}$, Raimundo Martins Filho ${ }^{5}$, Rildênio Renato Cavalcante ${ }^{2}$, João Batista Lopes $^{3}$, Maria Elizabete de Oliveira ${ }^{3}$}

\author{
1 Parte da dissertação apresentada pelo primeiro autor ao Programa de Mestrado em Ciência Animal - CCA-UFPI. \\ 2 Mestrando em Ciência Animal - CCA/UFPI. \\ 3 Departamento de Zootecnia - CCA/UFPI, CEP: 64049-550, Campus da Socopo S/N. \\ ${ }^{4}$ Embrapa Meio-Norte. Bolsista DCR - CNPq - FAPEPI. \\ ${ }^{5}$ Bolsista DCR - CNPq - FAPEPI vinculado ao Departamento de Zootecnia - CCA/UFPI.
}

RESUMO - Objetivou-se com esta pesquisa caracterizar fenotipicamente (peso e morfometria corporal) a conformação zootécnica de ovinos Santa Inês, machos e fêmeas de diferentes idades, criados em Teresina e Campo Maior, Piauí. Foram utilizados dados de pesos e de medidas corporais de 1.113 animais criados a campo e de 824 animais que participaram de exposição em feira agropecuária de Teresina. As características estudadas, comparadas pelo teste de Student-Newman-Keuls, foram: peso do animal, altura na cernelha e na garupa, comprimento corporal e de garupa, perímetro torácico e dois índices indicadores de capacidade corporal do animal. O efeito do manejo mostrou-se uma importante fonte de variação nas características avaliadas e o dimorfismo sexual foi mais acentuado nos animais mais velhos. O peso corporal foi mais influenciado pelos efeitos considerados nas análises que as demais medidas morfométricas. O índice que relaciona o peso ao comprimento corporal evidenciou que a tendência de acúmulo de gordura nos machos adultos é maior que nas fêmeas. O peso e as medidas morfométricas avaliadas apresentaram-se positivamente correlacionados, indicando a possibilidade de respostas correlacionadas se utilizados em programa de seleção da raça.

Palavras-chave: correlação, índice corporal, morfometria corporal, peso, tamanho

\section{Morphometric characterization of Santa Inês sheep raised in the regions of Teresina and Campo Maior, Piauí}

\begin{abstract}
This study aimed to carry out the phenotypic characterization of males and females of Santa Inês hair sheep through body weight measurements and morphmetric and conformation evaluations at different ages. Data consisted of body weights and measurements from 1,113 animals raised in Teresina and Campo Maior regions of Piauí State under pasture regime and 824 animals that participated in Agricultural Fairs in Teresina. Adjusted means for body weight, withers and croup height, corporal and croup length, thoracic perimeter and two indexes of body capacity of the animal were compared by the Student-Newman-Keuls test. The management effect was an important source of variation for the evaluated traits and the sexual dimorphism was more accentuated in the oldest animals. The effects included in the model influenced more the body weights than the morph metric measurements. The index of body capacity (weight and length) indicated a trend for more accumulation of fat in oldest males than in females. The body weight and the morphmetric measurements were positively correlated indicating the possibility of correlated response in breeding programs for the Santa Inês breed.
\end{abstract}

Key Words: body index, correlation, morphmetric, size, weight

\section{Introdução}

Nas regiões tropicais, tem-se procurado a melhoria da produtividade dos ovinos por meio da forma que parece ser a mais simples: a introdução de raças com bom desempenho em outras regiões (Sousa \& Morais, 2000). No entanto, a viabilidade desse procedimento não tem sido investigada e, como agravante, a conformação corporal dos descendentes de animais importados, especificamente no que tange a medidas corporais que envolvam dimensões e suas relações, não tem sido estudada no novo ambiente. Dessa forma, não se sabe se o tamanho corporal dos animais interage de forma eficiente com o novo ambiente e se esta interação resulta em níveis de produção aceitáveis, com mínima alteração no ambiente de produção.

Constata-se na literatura concordância entre pesquisadores quanto a não existir, do ponto de vista zootécnico, uma raça superior às demais para a produção de carne em 
todos os sistemas de criação, quer em grandes ou pequenos ruminantes (Winkler et al., 1997). O mesmo se aplica ao tamanho do animal: não se sabe se o maior ou o menor porte determinam maior produtividade. Há, entretanto, o consenso de que existe um tipo ou um tamanho mais adaptado às condições específicas de criação, assim como a afirmação de que animais que amadurecem mais cedo têm menor probabilidade de atingir peso adulto mais elevado em relação àqueles que amadurecem tardiamente (McManus et al., 2003).

Considerando que os sistemas de criação e as exigências de mercado são dinâmicos, a busca do tamanho ideal do animal para produção de carne é contínua. Nesse sentido, deve-se ressaltar que a lucratividade deve nortear as decisões sobre o tamanho ideal para determinada condição de ambiente ou mercado, pois o peso do animal e, conseqüentemente, o custo de mantença e produção serão diretamente influenciados.

Atualmente, não se dispõe de base científica para indicar o tamanho ideal de ovino de corte para a Região Nordeste, porém, já existe a consciência de que o animal Sem Raça Definida (SRD), além de não responder financeiramente a investimentos em tecnologia, não atende às exigências de qualidade de carne para competir com o produto importado e, por isso, sua exploração se mantém restrita às criações de subsistência ou extrativistas. O uso de cruzamentos tem sido proposto como solução para compatibilizar produtividade com adaptabilidade nesses ambientes (EMBRAPA, 1993).

O desenvolvimento, no Brasil, de raças deslanadas regionais como a Santa Inês apresenta-se como alternativa interessante para melhora da eficiência dos sistemas de produção de carne ovina. Segundo Sousa \& Morais (2000), o tamanho corporal do ovino adulto dessa raça tem sido motivo de propaganda em exposições agropecuárias, como se representasse uma grande vantagem. O maior tamanho corporal à idade adulta pode levar a raça a maiores requerimentos nutricionais e ao comprometimento de sua eficiência reprodutiva em condições de maior escassez alimentar. Diante desses argumentos, épreciso estabelecer o tamanho ideal para a raça, não somente em condições de semi-árido do Nordeste, mas também em outras regiões do país.

Nessa perspectiva, objetivou-se caracterizar fenotipicamente, quanto ao peso e à morfometria corporal, a conformação zootécnica de ovinos da raça Santa Inês explorados em diferentes sistemas de criação no Piauí e relacionar o tamanho do animal ao manejo utilizado no sistema de produção de carne.

\section{Material e Métodos}

Foram utilizados dados de registros de peso e medidas corporais de ovinos da raça Santa Inês, em diferentes idades e de ambos os sexos, dos quais 1.113 foram criados a campo, em fazendas das microrregiões de Teresina e Campo Maior, Piauí, e 824 animais participantes de julgamentos da raça em exposições agropecuárias em Teresina.

A microrregião de Campo Maior apresenta clima tropical seco, com precipitação pluviométrica média anual de $1.200 \mathrm{~mm}$, distribuída em sua maior parte no período de janeiro a maio, e temperatura média anual de 27 a $33^{\circ} \mathrm{C}$. A pastagem nativa explorada pela agropecuária local integra um complexo caracterizado por amplas extensões de vegetação herbácea, formando campos abertos de gramíneas e leguminosas, interrompidos por pequenas áreas esparsas de vegetação arbustivo-arbórea (Jacomine et al., 1986). A microrregião de Teresina apresenta condições climáticas similares, entretanto, a vegetação é bem distinta, com predominância de floresta mista, conhecida como mata de babaçu (Nascimento et al., 1981).

Os dados de peso e medidas corporais de animais participantes de julgamento em feiras agropecuárias de Teresina foram fornecidos pela Associação de Criadores de Caprinos e Ovinos do Piauí - APICCOVI e, nas análises, foram classificados como de nível alto de tecnologia de criação. Os dados de animais criados a campo foram obtidos em 17 propriedades, 11 da microrregião de Teresina e seis da microrregião de Campo Maior. Essas propriedades foram agrupadas em níveis baixo e médio de tecnologia de criação, considerando-se para essa classificação as observações in locoe as informações fornecidas pelo proprietário quanto ao sistema de criação adotado, como descrito a seguir:

nível baixo - criação em pastagem nativa, sem suplementação alimentar;

nível médio - criação em pastagem nativa e cultivada, com suplementação alimentar na entressafra.

A ausência de escrituração zootécnica na maioria das propriedades pesquisadas implicou na necessidade de avaliação da cronologia dentária, conforme descrito por Ribeiro (1998), para agrupar os animais por idade mais aproximada, formando-se as classes: dente-de-leite, $1 \underline{\underline{a}}$ e $2 \underline{a}$ mudas; e acima de 3 a muda.

As características avaliadas foram o peso corporal do animal $(\mathrm{kg})$, as alturas de cernelha e de garupa $(\mathrm{cm})$, os comprimentos corporal e de garupa $(\mathrm{cm})$ e o perímetro torácico $(\mathrm{cm})$.

Os pesos e as medidas morfométricas dos animais participantes de julgamento nas exposições agropecuárias 
foram obtidos do banco de dados da Associação de Criadores de Caprinos e Ovinos do Estado do Piauí e submetidos a análises para descarte de informações incompatíveis com a idade e de animais com duas ou mais medidas perdidas. Para os animais das fazendas pesquisadas, essas informações foram obtidas por um mesmo avaliador, com as mensurações realizadas sempre pela manhã.

As mensurações de morfometria corporal foram realizadas com o auxílio de fita métrica, com o animal mantido em posição correta de aprumos. A altura de cernelha foi medida entre o ponto mais alto da região interescapular e o solo e a altura na garupa, entre a tuberosidade sacral do ílio e o solo. O perímetro torácico foi medido na circunferência externa da cavidade torácica, junto às axilas. O comprimento corporal foi medido da parte cranial da tuberosidade maior do úmero até a parte caudal da tuberosidade isquiática e o comprimento de garupa entre a parte cranial da tuberosidade ilíaca e a caudal da tuberosidade isquiática.

Além do peso e das medidas morfométricas descritas, foram avaliados dois índices indicadores da capacidade corporal dos animais: a capacidade corporal (CC1), correspondente ao quociente entre o peso $(\mathrm{kg})$ e o comprimento corporal $(\mathrm{cm})$ do animal; e a capacidade corporal $(\mathrm{CC} 2)$, que correspondeu ao quociente entre o peso $(\mathrm{kg})$ e o perímetro torácico $(\mathrm{cm})$ do animal.

A análise estatística dos dados foi realizada utilizando-se o Método de Quadrados Mínimos, por meio do procedimento GLM do software Statistical Analysis System (SAS, 1990). Inicialmente, efetuou-se a análise de variância considerando-se grupos de contemporâneos no modelo, formados com a inclusão de microrregião, fazenda e ano da exposição, utilizando-se delineamento inteiramente casualizado, em um arranjo fatorial 3 x 2 (três classes de idade e dois sexos).

Face às particularidade dos dados (parte foi originada de banco de dados de exposições agropecuárias e parte coletada em fazendas), a influência do manejo de criação dos animais foi abordada de forma direta em uma segunda análise, realizada em um arranjo fatorial 3 × 3 × 2 (três níveis de tecnologia de criação, três classes de idade e dois sexos), representado pela fórmula:

$$
\begin{gathered}
\mathrm{Y}_{\mathrm{ijlm}}=\mu+\mathrm{NT}_{\mathrm{i}}+\mathrm{CI}_{\mathrm{j}}+\mathrm{S}_{1}+\left(\mathrm{NT} \mathrm{CI}_{\mathrm{ij}}+(\mathrm{NT} * \mathrm{~S})_{\mathrm{il}}+\left(\mathrm{CI} * \mathrm{~S}_{\mathrm{j} 1}\right.\right. \\
+\left(\mathrm{NT} * \mathrm{CI} \mathrm{S}_{\mathrm{ijl}}+\mathrm{e}_{\mathrm{ijlm}}\right.
\end{gathered}
$$

em que $\mathrm{Y}_{\mathrm{ijlm}}$ = resposta observada do nível de tecnologia de criação $i$, da classe de idade j e do sexo $1 ; \mu$ = média geral; $\mathrm{NT}_{\mathrm{i}}=$ efeitos do nível de tecnologia de criação i ( $\mathrm{i}=1$ a 3 ); $\mathrm{CI}_{\mathrm{j}}=$ efeitos da classe de idade $\mathrm{j}(\mathrm{j}=1$ a 3$) ; \mathrm{S}_{\mathrm{l}}=$ efeitos do sexo 1 (macho ou fêmea); $\left(\mathrm{NT}^{*} \mathrm{CI}\right)_{\mathrm{ij}}$ e $\left(\mathrm{NT}^{*} \mathrm{~S}\right)_{\mathrm{il}}=$ efeitos das interações nível de tecnologia $\times$ classe de idade e nível de tecnologia $\times$ sexo, respectivamente; $\left(\mathrm{CI}^{*} \mathrm{~S}\right)_{\mathrm{jl}}=$ efeitos da interação classe de idade $\times$ sexo; $(\mathrm{NT} * \mathrm{CI} * \mathrm{~S})_{\mathrm{ijl}}=$ efeitos da interação tripla da tecnologia de criação $\times$ classe de idade $\times$ sexo; e $e_{i j l m}=$ erro experimental. Nessa análise, a interação tripla foi significativa para todas as características e, portanto, optou-se por apresentar os dados considerando efeitos de nível de tecnologia e de classe de idade dentro de sexo.

As médias foram comparadas pelo teste StudentNewman-Keuls a $1 \%$ de probabilidade. Foram realizadas ainda análises de correlações simples entre as características pelo procedimento PROC COR.

\section{Resultados e Discussão}

Como demonstrado na Tabela 1, houve maior contribuição de fêmeas, pois, dos 1.937 registros avaliados, apenas $28,5 \%$ foram compostos de informações de machos, o que representa, na amostra de cada rebanho, uma proporção de mais de duas fêmeas para cada macho, destacando-se principalmente a redução da quantidade de machos adultos nos rebanhos.

As interações e os efeitos fixos apresentados no modelo foram significativos $(\mathrm{P}<0,01)$. A classe de idade, entre os efeitos estudados, foi o fator individual de maior causa de variação sobre as características, observando-se que a influência do sexo se acentuou com o aumento da idade.

Nessa análise os resultados representaram a média de valores obtidos de animais criados extensiva e intensivamente. Assim, os valores dos coeficientes de variação representaram, além dos erros aleatórios inerentes às formas de mensurações usadas, também um componente decorrente das diferenças de manejo entre as fazendas, não removíveis com o uso de grupos de contemporâneos.

No conjunto de dados avaliado, a maior variação foi observada para o peso corporal, seguido pelo perímetro torácico. O peso corporal apresentou coeficiente de variação de $21,77 \%$, indicando ser, entre as características avaliadas, a mais sujeita a influência de efeitos ambientais (Winkler et al., 1997). As medidas morfométricas, no entanto, apresentaram valores inferiores a $9 \%$, refletindo pequena influência de efeitos não considerados no modelo, como constataram Northcutt et al. (1992). Esses resultados divergem dos obtidos em bovinos por Lisboa \& Fernandes (1988), que afirmaram ser necessário número elevado de informações para avaliação de medidas morfométricas de animais a campo, em razão da dificuldade de mensuração precisa nos animais mais agressivos. 
Tabela 1 - Número de animais $(\mathrm{N})$, médias e desvios-padrão do peso corporal (kg), da altura na cernelha e na garupa (cm), do comprimento corporal e da garupa $(\mathrm{cm})$, do perímetro torácico $(\mathrm{cm})$ e dos índices de capacidade corporal (CC1 e CC2) ${ }^{\star *}$ de ovinos Santa Inês

Table 1 - Number of animals $(\mathrm{N})$, means and standard deviations for body weight ( $\mathrm{kg}$ ), withers and rump height (cm), body length (cm), thoracic perimeter (cm), rump length (cm) and body capacity indexes (CC1 and CC2) of Santa Inês sheep

\begin{tabular}{|c|c|c|c|c|c|c|c|c|c|c|}
\hline $\begin{array}{l}\text { Classe } \\
\text { de idade } \\
\text { Age class }\end{array}$ & $\begin{array}{c}\text { Sexo } \\
\text { Sex }\end{array}$ & $\mathrm{N}^{1}$ & $\begin{array}{l}\text { Peso }(\mathrm{kg}) \\
\text { Body weight }\end{array}$ & $\begin{array}{c}\text { Altura de } \\
\text { cernelha }(\mathrm{cm}) \\
\text { Withers height }\end{array}$ & $\begin{array}{c}\text { Altura de } \\
\text { garupa }(\mathrm{cm}) \\
\text { Rump height }\end{array}$ & $\begin{array}{l}\text { Comprimento } \\
\text { corporal }(\mathrm{cm}) \\
\text { Bodylength }\end{array}$ & $\begin{array}{c}\text { Perímetro } \\
\text { torácico }(\mathrm{cm}) \\
\text { Thoracic perimeter }\end{array}$ & $\begin{array}{l}\text { Comprimento } \\
\text { garupa }(\mathrm{cm}) \\
\text { Rump length }\end{array}$ & $\mathrm{CC} 1$ & $\mathrm{CC} 2$ \\
\hline \multirow{3}{*}{$\begin{array}{l}\text { Acima da } \\
3 \text { a muda } \\
\text { After the } \\
3^{\text {rd }} \text { shift }\end{array}$} & $\begin{array}{l}\text { Macho } \\
\text { Male }\end{array}$ & 107 & $79,5 \pm 17,2^{\mathrm{a}}$ & $82,7 \pm 8,2^{\mathrm{a}}$ & $82,3 \pm 7,4^{\mathrm{a}}$ & $85,5 \pm 7,5^{\mathrm{a}}$ & $102,1 \pm 12,8^{\mathrm{a}}$ & $26,0 \pm 4,1^{\mathrm{a}}$ & $0,91 \pm 0,15^{\mathrm{a}}$ & $0,75 \pm 0,12^{\mathrm{a}}$ \\
\hline & & & & & & & & & & \\
\hline & $\begin{array}{l}\text { Fêmea } \\
\text { Female }\end{array}$ & 449 & $54,1 \pm 16,3^{\mathrm{c}}$ & $73,0 \pm 7,3^{\mathrm{c}}$ & $72,8 \pm 7,0^{\mathrm{c}}$ & $74,7 \pm 7,3^{\mathrm{c}}$ & $90,8 \pm 11,3^{\mathrm{b}}$ & $23,0 \pm 3,7^{\mathrm{b}}$ & $0,72 \pm 0,15^{\mathrm{b}}$ & $0,58 \pm 0,11^{\mathrm{c}}$ \\
\hline \multirow{2}{*}{$\begin{array}{l}1 \stackrel{\mathrm{a}}{\mathrm{e}} 22^{\mathrm{a}} \\
\text { mudas } \\
1^{\text {st }} \text { and } 2^{\text {nd }} \\
\text { shifts }\end{array}$} & $\begin{array}{l}\text { Macho } \\
\text { Male }\end{array}$ & 135 & $60,6 \pm 15,1^{\mathrm{b}}$ & $77,2 \pm 7,4^{b}$ & $76,9 \pm 7,0^{b}$ & $78,6 \pm 7,2^{b}$ & $91,7 \pm 10,0^{\mathrm{b}}$ & $23,5 \pm 2,1^{b}$ & $0,75 \pm 0,14^{\mathrm{b}}$ & $0,63 \pm 0,14^{\mathrm{b}}$ \\
\hline & $\begin{array}{l}\text { Fêmea } \\
\text { Female }\end{array}$ & 416 & $46,3 \pm 14,4^{\mathrm{d}}$ & $71,4 \pm 6,7^{\mathrm{d}}$ & $71,4 \pm 6,7^{\mathrm{d}}$ & $73,3 \pm 6,8^{c}$ & $84,6 \pm 8,8^{c}$ & $22,3 \pm 2,1^{\mathrm{c}}$ & $0,62 \pm 0,13^{\mathrm{c}}$ & $0,53 \pm 0,14^{\mathrm{d}}$ \\
\hline \multicolumn{2}{|c|}{$\begin{array}{l}\text { Coeficiente } \\
\text { de variação (\%) }\end{array}$} & 21,77 & 6,34 & 6,20 & 7,93 & 8,91 & 8,31 & 17,70 & 15,45 & \\
\hline \multicolumn{11}{|c|}{ Coefficient of variation (\%) } \\
\hline
\end{tabular}

1 Número de animais (Number of animals).

* Médias seguidas de mesma letra na coluna não diferem $(P<0,01)$ pelo teste Student-Newman-Keuls.

** Índices de capacidade corporal CC1 (peso pelo comprimento corporal) e CC2 (peso pelo perímetro torácico).

* Means followed by the same letter in the column do not differ $(P<0.01)$ by Student-Newman-Keuls test.

** Body capacity index CC1 (body weight/body length) and CC2 (body weight/thoracic perimeter).

Quanto aos efeitos de sexo e classe de idade, observou-se significância $(\mathrm{P}<0,01)$ tanto para o peso corporal quanto para as medidas morfométricas, de modo que os machos apresentaram valores maiores que os das fêmeas, como observado por McMannus \& Miranda (1997). O peso corporal foi mais variável que as demais características, mostrando-se mais influenciado pelo ambiente, como constatado por Santos (2000).

Os machos adultos da raça Santa Inês apresentaram peso elevado, com aumento correspondente a quase $100 \%$ do peso dos animais dente-de-leite, idade recomendada para abate com carne de melhor qualidade (Sainz, 2000). O elevado peso à idade adulta indica a possibilidade de acúmulo excessivo de gordura, que contribui para redução da qualidade da carcaça e obtenção de animais mais tardios (Siqueira et al., 2001). Além disso, esse incremento pode não representar, obrigatoriamente, potencial de carne, o que justifica não recomendar o abate tardiamente.

Incrementos inferiores a $20 \%$ observados nos comprimentos corporal e de garupa e nas alturas de cernelha e de garupa à medida que aumenta a idade do animal são considerados indicativos de diferença na velocidade de cresci- mento ósseo em relação aos aumentos em peso na carcaça. Nessa afirmação, está implícito que o acúmulo de gordura, com o aumento da idade, constitui-se um componente importante do ganho de peso da carcaça, como referenciado por Bueno et al. (1999) e Sainz (2000).

O dimorfismo sexual na raça Santa Inês, nas características avaliadas, acentuou-se à medida que os animais foram envelhecendo, destacando-se a superioridade dos machos, como observado por McManus \& Miranda (1997) e Sainz (2000). Quanto ao comprimento de garupa, houve diferença significativa, de modo que as fêmeas da classe dente-deleite foram aproximadamente $6 \%$ superiores aos machos. A superioridade dos machos para as características morfométricas, considerando os dados na literatura, pode ser atribuída à atividade hormonal, como ocorre em eqüinos (Miserani et al., 2001).

O aumento nos valores do índice de capacidade corporal (relaciona o peso ao perímetro torácico do animal (CC2) à medida que envelhece), independentemente do sexo e da classe de idade, foi proporcionalmente menor que o observado para o índice que relaciona o peso ao comprimento corporal (CC1). Esse comportamento indica, em parte, que 
a partir de determinada idade o crescimento ósseo do animal parou, mas o peso corporal continuou a aumentar, enquanto o peso e o perímetro torácico parecem crescer simultaneamente por mais tempo.

Segundo Bueno et al. (1999), não há mais variação considerável no comprimento corporal quando o animal atinge a maturação esquelética. Portanto, quando findou o crescimento ósseo dos animais adultos, o acréscimo no valor do índice CC1 ocasionou deposição de músculo e gordura. Com a metodologia utilizada não foi possível detectar esse ponto, mas, conforme demonstrado na literatura, a intensificação de deposição de gordura no animal adulto é maior. Assim, considerando a facilidade de obtenção desse índice, seria interessante determinar seu valor para a raça Santa Inês para caracterizar a idade de abate. Outro aspecto importante é que pode não ocorrer proporcionalidade entre os índices avaliados, pois existe variação entre animais quanto à velocidade de crescimento esquelético e à velocidade de deposição de tecido adiposo, como afirmam Sainz (2000) e Siqueira (2000).

Nas Tabelas 2 e 3 são apresentadas, respectivamente, para machos e fêmeas das diferentes classes de idades, as médias e os desvios-padrão para peso e medidas corporais avaliadas, comparados pelo teste de Student-NewmanKeuls a $1 \%$ de probabilidade. Os resultados foram obtidos com a substituição, no modelo, do grupo de contemporâneo pelo nível de tecnologia de criação, sendo a análise realizada em um fatorial triplo, no qual todos os efeitos fixos e interações constantes no modelo foram significativos $(\mathrm{P}<0,05)$.

O potencial de crescimento da raça e os efeitos dos manejos de alta ou baixa tecnologia (Tabelas 2 e 3, para machos e fêmeas, respectivamente) foram analisados dentro de sexo. Os pesos corporais superiores a 100 e $80 \mathrm{~kg}$, respectivamente, para machos e fêmeas adultos no nível de alta tecnologia, foram similares aos valores apresentados por Sousa et al. (2003) e reforçam a afirmação de Sousa \& Morais (2000) de que o alto tamanho corporal na raça Santa Inês tem sido objeto de propaganda, mas pode resultar em maior requerimento nutricional para mantença.

Observaram-se valores médios de 47 e 107 kg para peso corporal dos machos adultos nos níveis de baixa e alta tecnologia de criação, respectivamente, o que corresponde a um acréscimo de $127 \%$. Na classe dente-de-leite, a variação de peso foi, na mesma ordem, de 30,4 a 59,9 kg. Nas fêmeas, a variação, apesar de menor, teve o mesmo comportamento, refletindo bem o efeito do manejo dos animais de exposições agropecuárias em relação ao realizado nas fazendas da região. O peso corporal dos animais criados a campo equiparou-se aos resultados obtidos por Araújo et

Tabela 2 - Médias e desvios-padrão do peso corporal (kg), das alturas na cernelha e na garupa (cm), dos comprimentos corporal e da garupa $(\mathrm{cm})$, do perímetro torácico $(\mathrm{cm})$ e dos índices de capacidade corporal ${ }^{\star *}(\mathrm{CC} 1$ e CC2) de ovinos Santa Inês machos de diferentes classes de idade, em análise com os dados agrupados por nível de tecnologia de criação

Table 2 - Means and standard deviations for body weight, withers height, rump height, body length, thoracic perimeter, rump length (cm) and body capacity indexes (CC1 and CC2) of Santa Inês males of different classes of age from analyses of data grouped by management level

\begin{tabular}{lccccccc}
\hline $\begin{array}{l}\text { Nível de } \\
\text { tecnologia }\end{array}$ & $\begin{array}{c}\text { Peso }(\mathrm{kg}) \\
\text { Bodyweight }\end{array}$ & $\begin{array}{c}\text { Altura de } \\
\text { cernelha }(\mathrm{cm}) \\
\text { Wathersheight }\end{array}$ & $\begin{array}{c}\text { Altura de } \\
\text { garupa }(\mathrm{cm}) \\
\text { Rumpheight }\end{array}$ & $\begin{array}{c}\text { Comprimento } \\
\text { corporal }(\mathrm{cm})\end{array}$ & $\begin{array}{c}\text { Perímetro } \\
\text { torácico }(\mathrm{cm})\end{array}$ & $\begin{array}{c}\text { Comprimento } \\
\text { Barupa }(\mathrm{cm})\end{array}$ & CC1 \\
Thentheracic perimeter & Rump length & CC2 \\
\hline
\end{tabular}

Acima da 3 a muda

After the $3^{\text {rd }}$ shift

\begin{tabular}{|c|c|c|c|c|c|c|c|c|}
\hline Alto (high) & $107,6 \pm 21,7^{\mathrm{a}}$ & $84,4 \pm 6,6^{b}$ & $84,1 \pm 6,2^{\mathrm{a}}$ & $86,6 \pm 6,6^{\mathrm{b}}$ & $117,0 \pm 13,4^{\mathrm{a}}$ & - & $1,24 \pm 0,19^{\mathrm{a}}$ & $0,92 \pm 0,10^{\mathrm{a}}$ \\
\hline Médio (medium) & $83,9 \pm 20,5^{b}$ & $88,3 \pm 9,7^{\mathrm{a}}$ & $86,4 \pm 8,7^{\mathrm{a}}$ & $90,8 \pm 7,8^{\mathrm{a}}$ & $104,8 \pm 12,8^{b}$ & $28,0 \pm 4,1^{\mathrm{a}}$ & $0,92 \pm 0,12^{\mathrm{b}}$ & $0,79 \pm 0,11^{b}$ \\
\hline Baixo (low) & $47,0 \pm 18,1^{\mathrm{c}}$ & $75,3 \pm 5,7^{\mathrm{c}}$ & $76,3 \pm 6,4^{b}$ & $79,0 \pm 6,6^{\mathrm{b}}$ & $84,7 \pm 9,2^{\mathrm{c}}$ & $24,0 \pm 2,9^{\mathrm{b}}$ & $0,58 \pm 0,11^{\mathrm{c}}$ & $0,54 \pm 0,10^{\mathrm{c}}$ \\
\hline
\end{tabular}

1ㅡㅡ e $2 \underline{\mathrm{a}}$ mudas

$1^{\text {st }}$ and $2^{\text {nd }}$ shifts

\begin{tabular}{|c|c|c|c|c|c|c|c|c|}
\hline Alto (high) & $88,6 \pm 17,0^{\mathrm{a}}$ & $81,5 \pm 6,5^{\mathrm{a}}$ & $81,5 \pm 5,4^{\mathrm{a}}$ & $82,7 \pm 6,3^{\mathrm{a}}$ & $107,1 \pm 11,3^{\mathrm{a}}$ & - & $1,07 \pm 0,21^{\mathrm{a}}$ & $0,82 \pm 0,13^{\mathrm{a}}$ \\
\hline Médio (medium) & $60,6 \pm 18,4^{b}$ & $80,0 \pm 5,9^{a}$ & $78,7 \pm 5,8^{b}$ & $82,7 \pm 6,0^{a}$ & $92,3 \pm 9,3^{\mathrm{b}}$ & $25,6 \pm 3,6^{\mathrm{a}}$ & $0,71 \pm 0,16^{\mathrm{b}}$ & $0,64 \pm 0,14^{\mathrm{b}}$ \\
\hline Baixo (low) & $32,7 \pm 14,5^{\mathrm{c}}$ & $70,0 \pm 5,4^{\mathrm{b}}$ & $70,3 \pm 5,3^{c}$ & $70,5 \pm 6,1^{b}$ & $75,7 \pm 9,1^{c}$ & $21,5 \pm 2,5^{b}$ & $0,46 \pm 0,14^{\mathrm{c}}$ & $0,43 \pm 0,10^{\mathrm{c}}$ \\
\hline
\end{tabular}

Dente-de-leite

Baby tooth

\begin{tabular}{|c|c|c|c|c|c|c|c|c|}
\hline Alto (high) & $59,9 \pm 13,3^{\mathrm{a}}$ & $73,8 \pm 5,4^{\mathrm{a}}$ & $74,6 \pm 5,6^{\mathrm{a}}$ & $73,5 \pm 6,3^{\mathrm{a}}$ & $91,7 \pm 9,2^{\mathrm{a}}$ & - & $0,81 \pm 0,15^{\mathrm{a}}$ & $0,64 \pm 0,09^{\mathrm{a}}$ \\
\hline Médio (medium) & $35,0 \pm 12,6^{b}$ & $68,2 \pm 5,1^{b}$ & $68,1 \pm 4,9^{b}$ & $67,6 \pm 6,0^{b}$ & $75,9 \pm 8,3^{b}$ & $21,6 \pm 2,0^{\mathrm{a}}$ & $0,52 \pm 0,14^{b}$ & $0,45 \pm 0,14^{\mathrm{b}}$ \\
\hline Baixo (low) & $30,4 \pm 12,8^{b}$ & $69,1 \pm 4,8^{b}$ & $68,1 \pm 4,7^{\mathrm{b}}$ & $67,2 \pm 6,0^{b}$ & $74,5 \pm 8,5^{b}$ & $19,6 \pm 2,4^{b}$ & $0,44 \pm 0,13^{b}$ & $0,40 \pm 0,11^{\mathrm{b}}$ \\
\hline
\end{tabular}

* Médias seguidas de mesma letra na coluna não diferem $(P<0,01)$ pelo teste de Student-Newman-Keuls.

** Índices de capacidade corporal CC1 (peso/comprimento corporal) e CC2 (peso/perímetro torácico).

* Means followed by the same letter in the column do not differ $(P<0.01)$ by Student-Newman-Keuls test.

** Body capacity indexes CC1 (body weight/body length) and CC2 (body weight/thoracic perimeter). 
Tabela 3 - Médias e desvios-padrão do peso corporal ( $\mathrm{kg}$ ), das alturas na cernelha e na garupa (cm), dos comprimentos corporal e da garupa $(\mathrm{cm})$, do perímetro torácico $(\mathrm{cm})$ e dos índices de capacidade corporal** $(\mathrm{CC} 1$ e CC2) de ovinos Santa Inês fêmeas de diferentes classes de idade, em análise com os dados agrupados por nível de tecnologia de criação

Table 3 - Means and standard deviations for body weight, withers height, rump height, body length, thoracic perimeter, rump length and body capacity indexes (CC1 and CC2) of Santa Inês, females of different classes of age from analyses of data grouped by management level

\begin{tabular}{|c|c|c|c|c|c|c|c|c|}
\hline $\begin{array}{l}\text { Nível de } \\
\text { tecnologia } \\
\text { Management level }\end{array}$ & $\begin{array}{l}\text { Peso }(\mathrm{kg}) \\
\text { Body weight }\end{array}$ & $\begin{array}{c}\text { Altura de } \\
\text { cernelha }(\mathrm{cm}) \\
\text { Withers height }\end{array}$ & $\begin{array}{l}\text { Altura de } \\
\text { garupa }(\mathrm{cm}) \\
\text { Rump height }\end{array}$ & $\begin{array}{c}\text { Comprimento } \\
\text { corporal }(\mathrm{cm}) \\
\text { Bodylength }\end{array}$ & $\begin{array}{c}\text { Perímetro } \\
\text { torácico }(\mathrm{cm}) \\
\text { Thoracic perimeter }\end{array}$ & $\begin{array}{l}\text { Comprimento } \\
\text { garupa }(\mathrm{cm}) \\
\text { Rump length }\end{array}$ & CC1 & $\mathrm{CC} 2$ \\
\hline
\end{tabular}

A partir da 3 a muda

More than $3^{\text {rd }}$ change

\begin{tabular}{|c|c|c|c|c|c|c|c|c|}
\hline Alto $(h i g h)$ & $81,3 \pm 18,1^{\mathrm{a}}$ & $75,2 \pm 7,6^{\mathrm{a}}$ & $76,4 \pm 7,0^{\mathrm{a}}$ & $76,9 \pm 5,8^{\mathrm{a}}$ & $107,5 \pm 12,4^{\mathrm{a}}$ & - & $1,05 \pm 0,17^{\mathrm{a}}$ & $0,75 \pm 0,09^{a}$ \\
\hline Médio (medium) & $46,6 \pm 16,1^{b}$ & $74,7 \pm 7,8^{\mathrm{a}}$ & $73,8 \pm 6,8^{b}$ & $77,6 \pm 6,2^{\mathrm{a}}$ & $86,4 \pm 11,6^{\mathrm{b}}$ & $24,4 \pm 3,8^{\mathrm{a}}$ & $0,92 \pm 0,14^{\mathrm{b}}$ & $0,53 \pm 0,09^{\mathrm{b}}$ \\
\hline $\begin{array}{l}\text { Baixo (low) } \\
1^{\mathrm{a}} \text { e } 2^{-} \text {muda } \\
\left(1^{\text {st }} \text { and } 2^{\text {nd }} \text { changes }\right)\end{array}$ & $34,6 \pm 15,7^{\mathrm{c}}$ & $69,0 \pm 7,0^{\mathrm{b}}$ & $68,2 \pm 6,9^{c}$ & $69,5 \pm 6,0^{b}$ & $78,4 \pm 10,4^{\mathrm{c}}$ & $21,7 \pm 3,1^{\mathrm{b}}$ & $0,50 \pm 0,10^{\mathrm{c}}$ & $0,44 \pm 0,10^{\mathrm{c}}$ \\
\hline $\begin{array}{l}\text { Alto (high) } \\
\text { Médio (medium) } \\
\text { Baixo (low) } \\
\text { Dente-de-leite } \\
\text { Milk tooth }\end{array}$ & $\begin{array}{l}70,0 \pm 17,0^{\mathrm{a}} \\
39,8 \pm 14,0^{\mathrm{b}} \\
32,7 \pm 12,5^{\mathrm{c}}\end{array}$ & $\begin{array}{l}75,0 \pm 6,9^{\mathrm{a}} \\
72,5 \pm 6,7^{\mathrm{b}} \\
66,8 \pm 6,4^{\mathrm{c}}\end{array}$ & $\begin{array}{l}76,2 \pm 7,3^{\mathrm{a}} \\
71,9 \pm 7,1^{\mathrm{b}} \\
66,2 \pm 6,9^{\mathrm{c}}\end{array}$ & $\begin{array}{l}76,5 \pm 5,4^{\mathrm{a}} \\
74,5 \pm 5,0^{\mathrm{b}} \\
68,9 \pm 5,1^{\mathrm{c}}\end{array}$ & $\begin{array}{c}99,1 \pm 11,7^{\mathrm{a}} \\
80,8 \pm 9,2^{\mathrm{b}} \\
74,1 \pm 9,0^{\mathrm{c}}\end{array}$ & $\begin{array}{c}- \\
23,5 \pm 3,4^{\mathrm{a}} \\
21,2 \pm 3,1^{\mathrm{b}}\end{array}$ & $\begin{array}{l}0,92 \pm 0,17^{\mathrm{a}} \\
0,71 \pm 0,12^{\mathrm{b}} \\
0,42 \pm 0,11^{\mathrm{c}}\end{array}$ & $\begin{array}{l}0,71 \pm 0,10^{\mathrm{a}} \\
0,49 \pm 0,06^{\mathrm{b}} \\
0,39 \pm 0,07^{\mathrm{c}}\end{array}$ \\
\hline $\begin{array}{l}\text { Alto (high) } \\
\text { Médio (medium) } \\
\text { Baixo (low) }\end{array}$ & $\begin{array}{l}49,4 \pm 15,3^{\mathrm{a}} \\
40,1 \pm 14,0^{\mathrm{b}} \\
27,4 \pm 11,2^{\mathrm{c}}\end{array}$ & $\begin{array}{l}70,3 \pm 6,9^{\mathrm{b}} \\
72,1 \pm 7,3^{\mathrm{a}} \\
65,8 \pm 4,8^{\mathrm{c}}\end{array}$ & $\begin{array}{l}71,5 \pm 7,0^{\mathrm{a}} \\
71,7 \pm 7,2^{\mathrm{a}} \\
65,8 \pm 6,1^{\mathrm{b}}\end{array}$ & $\begin{array}{l}68,6 \pm 5,4^{\mathrm{b}} \\
74,2 \pm 5,7^{\mathrm{a}} \\
65,3 \pm 5,2^{\mathrm{b}}\end{array}$ & $\begin{array}{c}87,8 \pm 10,2^{\mathrm{a}} \\
80,3 \pm 10,0^{\mathrm{b}} \\
70,0 \pm 8,9^{\mathrm{c}}\end{array}$ & $\begin{array}{c}- \\
23,0 \pm 3,0^{\mathrm{a}} \\
20,5 \pm 3,0^{\mathrm{b}}\end{array}$ & $\begin{array}{l}0,72 \pm 0,14^{\mathrm{a}} \\
0,52 \pm 0,09^{\mathrm{b}} \\
0,42 \pm 0,10^{\mathrm{c}}\end{array}$ & $\begin{array}{l}0,56 \pm 0,04^{\mathrm{a}} \\
0,49 \pm 0,04^{\mathrm{b}} \\
0,39 \pm 0,05^{\mathrm{c}}\end{array}$ \\
\hline
\end{tabular}

* Médias seguidas de mesma letra na coluna não diferem $(P<0,01)$ pelo teste de Student-Newman-Keuls.

** Índices de capacidade corporal CC1 (peso/comprimento corporal) e CC2 (peso/perímetro torácico).

* Means followed by the same letter in the column do not differ $(P<0.01)$ by Student-Newman-Keuls test.

** Body capacity indexes CC1 (body weight/body length) and CC2 (body weight/thoracic perimeter).

al. (1996) e McManus et al. (2003), ressaltando-se, no entanto, que o manejo de mais baixa qualidade pode não ser adequado para que a raça expresse o potencial de crescimento. Por outro lado, no manejo de elevada tecnologia, as condições são favoráveis à deposição de gordura.

Destaca-se que a menor quantidade de machos adultos e o tratamento preferencial nos rebanhos do nível alto de tecnologia de criação criam viés para mais no peso adulto da raça. Ao mesmo tempo, para animais criados em ambientes quentes com elevado peso à idade adulta, são muitos os inconvenientes ao bem-estar e à funcionalidade (Rosa \& Lobo, 1998), sendo observados indicadores de desconforto no animal, como elevação da freqüência respiratória e permanência por longo período deitado, principalmente de fêmeas no final da gestação.

As características morfométricas foram igualmente afetadas pelas diferenças de qualidade do manejo das propriedades $(\mathrm{P}<0,01)$, representando considerável fonte de variação no tamanho do animal, principalmente quanto ao perímetro torácico. Observou-se certa proporcionalidade no incremento na variação dessas características, o que reforça a constatação de correlações entre essas características, tanto para ovinos como para os demais ruminantes (Silva, 1990).

O efeito do nível de tecnologia de criação sobre a conformação dos animais ficou bem evidente nesta pes- quisa. Para os animais adultos, os valores do índice de capacidade corporal (CC1) foram próximos a 0,5 e superiores à unidade, respectivamente, nos níveis de baixa e de alta tecnologia de criação. Na primeira situação, está caracterizada a necessidade de melhor manejo para que a raça expresse seu potencial de crescimento e não, obrigatoriamente, que apresente conformação longilínea. Por outro lado, os valores superiores à unidade evidenciam acúmulo de gordura, pois, segundo Bueno et al. (1999), Reis et al. (2001) e Garcia et al. (2003), ocorre redução na taxa de conversão alimentar para deposição de carne no animal de idade mais elevada.

Também ficou caracterizada a influência do sexo na conformação corporal, sendo que apenas as fêmeas mais velhas do nível de alta tecnologia de criação apresentaram índice CC1 superior a 1 (um), que é indesejável se indicar obesidade, considerando que, geralmente, matrizes obesas apresentam dificuldades reprodutivas.

Considerando que a conformação corporal dos animais é definida pelas relações entre a altura e o comprimento e a profundidade do corpo, que variam em função da raça e da idade, a caracterização da melhor conformação de animais da raça Santa Inês para as condições pesquisadas ganha importância, principalmente se identificado um índice de capacidade corporal com o qual se possa inferir se o predomínio de deposição de gordura seria este um bom critério de seleção. 
Tabela 4 - Correlações simples entre o peso corporal $(\mathrm{kg})$, as alturas na cernelha e na garupa (cm), os comprimentos corporal e de garupa $(\mathrm{cm})$, o perímetro torácico $(\mathrm{cm})$ e os índices de capacidade corporal* $(\mathrm{CC} 1$ e CC2) de ovinos Santa Inês

Table 4 - Correlation among body weight, withers and rump height, body length, thoracic perimeter, rump length and body capacity index (CC1 and CC2) of Santa Inês sheep

\begin{tabular}{|c|c|c|c|c|c|c|c|}
\hline $\begin{array}{l}\text { Característica } \\
\text { Trait }\end{array}$ & $\mathrm{AC}$ & AG & $\mathrm{CC}$ & CG & PT & $\mathrm{CC} 1$ & $\mathrm{CC} 2$ \\
\hline Peso corporal (Bodyweight) & 0,74 & 0,79 & 0,66 & 0,76 & 0,94 & 0,97 & 0,97 \\
\hline Altura de cernelha (AC) (Withers height) & & 0,94 & 0,77 & 0,76 & 0,72 & 0,65 & 0,73 \\
\hline Comprimento corporal (CC) (Body length) & & & & & 0,67 & 0,48 & 0,65 \\
\hline Perímetro torácico (PT) (Thoracic perimeter) & & & & & & 0,92 & 0,86 \\
\hline Comprimento na garupa (CG) (Rump length) & & & & & & 0,69 & 0,72 \\
\hline
\end{tabular}

* Índices de capacidade corporal CC1 (peso/comprimento corporal) e CC2 (peso/perímetro torácico)

* Body capacity indexes CC1 (body weight/body length) and CC2 (body weight/thoracic perimeter).

Valores de correlações positivas elevados foram constatados entre as medidas morfométricas e entre essas medidas e o peso corporal (Tabela 4), o que está de acordo com os resultados de várias pesquisas, tanto em ovinos como nos demais ruminantes (Razook et al., 1990; Fernandes et al., 1996; Scarpati et al., 1996; Cyrillo et al., 2000; Santos, 2000; Santana et al., 2001; Rocha et al., 2003). Esses resultados podem indicar também que resposta correlacionada nas demais características pode ocorrer se aquela de mais fácil mensuração for usada como critério de seleção (Badenhorst et al., 1991).

Portanto, a seleção de animais com pesos mais elevados à idade adulta pode resultar em aumento das medidas morfométricas e, conseqüentemente, do tamanho do animal. Em decorrência das altas correlações com o peso corporal, alguns autores (Araújo et al., 1996; Ledic \& Garcia Deragon, 1997) afirmaram que medidas morfométricas, prin cipalmente o perímetro torácico, podem ser utilizadas para determinar o peso do animal na ausência de balança.

O valor próximo da unidade constatado para as correlações entre os índices $(0,95)$ é uma indicação de similaridade do peso de animais longilíneos aos de maior profundidade corporal, o que impossibilita recomendar, para a região considerada, a seleção de animais com maior ou menor comprimento corporal. Entretanto, a maior correlação do índice CC2 com as alturas analisadas pode ser uma indicação de que os animais "pernaltas" não devem ser preferidos em programas de seleção.

\section{Conclusões}

O manejo de criação constituiu um importante fator de variação do peso corporal e das medidas morfométricas da raça na região, com modificação acentuada do porte dos animais entre condições distintas de criação.
A diferença entre machos e fêmeas, tanto para peso corporal como para medidas morfométricas, acentuou-se à medida que a idade dos animais aumentou.

$\mathrm{O}$ índice que relaciona o peso ao comprimento corporal evidenciou que a tendência de acúmulo de gordura nos machos adultos é maior que nas fêmeas.

As características peso e medidas morfométricas avaliadas mostraram-se positivamente correlacionadas, indicando a possibilidade de resposta correlacionada se utilizadas em programa de seleção da raça.

\section{Literatura Citada}

ARAÚJO, A.M.; VASCONCELOS, I.M.A.; SILVA, F.L.R. Medidas corporais de ovinos deslanados Santa Inês como indicadoras do peso vivo. Ciência Animal, v.6, n.1, p.64-68, 1996.

BADENHORST, M.A.; OLIVIER, J.J.; SCOEMAN, S.J. Ondersock in seleksiemaatsatawwe by Afrinoskape: genetic parameters in Afrinos sheep. South African Journal of Animal Science, v.31, n.3, p.162-165, 1991.

BUENO, M.S.; SANTOS, L.E.; CUNHA, E.A. et al. Avaliação de carcaças de cabritos abatidos com diferentes pesos vivos. Revista Nacional da Carne, n.272, p.72-79, 1999.

CYRILLO, J.N.S.G.; RAZOOK, A.G.; FIGUEIREDO, L.A. et al. Efeitos da seleção para peso pós-desmame sobre medidas corporais e perímetro escrotal de machos Nelore de Sertãozinho (SP). Revista Brasileira de Zootecnia, v.29, n.2, p.403412, 2000.

EMPRESA BRASILEIRA DE PESQUISA AGROPECUÁRIA EMBRAPA. Avaliação econômica e produtiva de dois sistemas de produção de ovinos de corte utilizando cruzamentos, em Sobral (CE). Sobral: EMBRAPA-CNPC, 1993. 35p. (Relatório de Projeto).

FERNANDES, A.; MAGNABOSCO, C.U.; OJALA, M. Estimativas de parâmetros genéticos e ambientes de medidas corporais e peso em bovinos da raça Brahman nos trópicos. In: REUNIÃO ANUAL DA SOCIEDADE BRASILEIRA DE ZOOTECNIA, 33. 1996, Fortaleza. Anais... Fortaleza: Sociedade Brasileira de Zootecnia, 1996. p.136-138.

GARCIA, C.A.; MONTEIRO, A.L.G.; COSTA, C. et al. Medidas objetivas e composição tecidual da carcaça de cordeiros alimentados com diferentes níveis de energia em creep feeding. Revista Brasileira de Zootecnia, v.32, n.6, p.1380-1390, 2003. 
JACOMINE, P.K.T.; CAVALCANTE, A.C.; PESSOA, S.C.P. Levantamento exploratório - reconhecimento de solos no Estado do Piauí. Rio de Janeiro: EMBRAPA - SNLCS; SUDENE - DRN, 1986.v.1, 678p. (EMBRAPA SNLS: Boletim de Pesquisa, 36; SUDENE: Série Recursos de Solos, 18).

LEDIC, I.L.; GARCIA DERAGON, L.A. Correlação fenotípica entre medidas corporais e peso em touros Nelore. Arquivo Brasileiro de Medicina Veterinária e Zootecnia, v.49, n.5, p.649654, 1997.

LISBOA, S.R.; FERNANDES, L.C.O. Efeito do tamanho corporal na fertilidade da primeira e segunda estação de monta, e na produtividade de fêmeas cruza Charolês. Revista Brasileira de Zootecnia, v.16, n.3, p.204-214, 1988.

McMANUS, C.; MIRANDA, R.M. Comparação das raças de ovinos Santa Inês e Bergamácia no Distrito Federal. Revista Brasileira de Zootecnia, v.26, n.5, p.1055-1059, 1997.

McMANUS, C.; EVANGELISTA, C.; FERNANDES, L.A.C. et al. Curvas de crescimento de ovinos Bergamácia criados no Distrito Federal. Revista Brasileira de Zootecnia, v.32, n.5, p.12071212, 2003

MISERANI, M.G.; McMANUS, C.; SANTOS, S.A. et al. Fatores que influem nas características lineares do cavalo Pantaneiro. In: REUNIÃO ANUAL DA SOCIEDADE BRASILEIRA DE ZOOTECNIA, 38., 2001, Piracicaba. Anais... Piracicaba: Sociedade Brasileira de Zootecnia, 2001. (CD-ROM).

NASCIMENTO, H.T.S.; NOVELLY, P.E.; RAMOS, G.M. Produtividade de pastagens nativa da "Zona de Mimoso" e da "Zona de Agreste". In: SEMINÁRIO DE PESQUISA AGROPECUÁRIA DO PIAUÍ, 2., 1980, Teresina. Anais... Teresina: EMBRAPA/UEPAE, 1981. p.145-51.

NORTHCUTT, S.L.; WILSON, D.E.; WILLHAM, R.L. Adjusting weight for body condition score in Angus cows. Journal of Animal Science, v.70, p.1342-1345, 1992.

RAZOOK, A.G.; LIMA, F.P.; BONILHA NETO, L.M. Respostas correlacionadas à seleção para peso pós-desmame sobre características morfológicas de bovinos Nelore. Boletim da Indústria Animal, v.46, p.246-257, 1990.

REIS, W.; JOBIN, C.C.; MACEDO, F.A.F. et al. Características de cordeiro alimentados com dietas contendo grãos de milho conservado de diferentes formas. Revista Brasileira de Zootecnia, v.30, n.4, p.1308-1315, 2001.

RIBEIRO, S.D. Caprinocultura: criação racional de caprinos. São Paulo: Nobel, 1998. 319p.

ROCHA, E.D.; ANDRADE, V.J.; EUCLIDES FILHO, K. et al. Tamanho de vacas Nelore adultas e seus efeitos no sistema de produção de gado de corte. Arquivo Brasileiro de Medicina Veterinária e Zootecnia, v.55, n.4, p.474-479, 2003.

ROSA, A.N.; LÔBO, R.B. Considerações sobre o tamanho adulto de matrizes nelore. In: SIMPÓSIO NACIONAL DE MElHORAMENTO ANIMAL, 2., 1998, Uberaba. Anais... Viçosa, MG: Universidade Federal de Viçosa, 1998. 465p.
SAINZ, R.D. Avaliação de carcaças e cortes comerciais de carne caprina e ovina. In: SINCORTE, 1., 2000, João Pessoa. Anais... João Pessoa: Empresa Estadual de Pesquisa Agropecuária da Paraíba, 2000. p.237-250.

SANTANA, A.F.S.; COSTA, G.B.; FONSECA, L.S. Correlações entre peso e medidas corporais em ovinos jovens da raça Santa Inês. Revista Brasileira de Produção Animal, v.1, n.1, p.74-77, 2001.

SANTOS, A.M. Caracterização morfométrica da Raça Simental em exposições agropecuárias do Brasil. Belém: Universidade Federal do Pará, 2000. 65p. Dissertação (Mestrado em Ciências Biológicas) - Universidade Federal do Pará, 2000.

SCARPATI, M.T.V.; MAGNABOSCO, C.U.; JOSAHKIAN, L.A. et al. Estudo de medidas corporais e peso vivo em animais jovens da Raça Nelore. In: REUNIÃO ANUAL DA SOCIEDADE BRASILEIRA DE ZOOTECNIA, 33., 1996, Fortaleza.Anais... Fortaleza: Sociedade Brasileira de Zootecnia, 1996. p.110-112.

STATISTICAL ANALYSIS SYSTEM - SAS. System for linear models. Cary: SAS Institute, 1990. 211p.

SILVA, F.L.R. Efeito de fatores genéticos e de ambiente sobre o desempenho de ovinos mestiços Santa Inês, no Estado do Ceará. Viçosa, MG: Universidade Federal de Viçosa, 1990 93p. Dissertação (Mestrado em Zootecnia) - Universidade Federal de Viçosa, 1990.

SIQUEIRA, E.R. Sistemas de confinamento de ovinos para corte do sudeste do Brasil. In:. SINCORTE, 1., 2000, João Pessoa. Anais... João Pessoa: Empresa Estadual de Pesquisa Agropecuária da Paraíba, 2000. p.107-117.

SIQUEIRA, E.R.; SIMÕES, C.D.; FERNANDES, S. Efeito do sexo e do peso ao abate sobre a produção de carne de cordeiro. Morfometria da carcaça, pesos dos cortes, composição tecidual e componentes não constituintes da carcaça. Revista Brasileira de Zootecnia, v.30, n.4, p.1299-1307, 2001.

SOUSA, W.H., MORAIS, O.R. Programa de melhoramento genético para ovinos deslanados do Brasil: ovinos da raça Santa Inês. In: SINCORTE, 1., 2000. João Pessoa. Anais... João Pessoa: Empresa Estadual de Pesquisa Agropecuária da Paraíba, 2000. p.223-229.

SOUSA, W.H.; LÔBO, R.N.B.; MORAIS, O.R. Ovinos Santa Inês: estado de arte e perspectivas. In: SIMPÓSIO INTERNACIONAL SOBRE CAPRINOS E OVINOS DE CORTE, 2., 2003, João Pessoa. Anais... João Pessoa: Empresa Estadual de Pesquisa Agropecuária da Paraíba, 2003. (CD-ROM)

WINKLER, R.; PENNA, V.M.; PEREIRA, C.S. et al. Estimativas de parâmetros genéticos e fenotípicos de peso e de medidas corporais em fêmeas bovinas adultas da raça Guzerá. Arquivo Brasileiro de Medicina Veterinária e Zootecnia, v.49, n.3, p.353-363, 1997.

Recebido: 08/03/05 Aprovado: 04/07/06 